\title{
Serum Brain-Derived Neurotrophic Factor Levels Are Associated with Skeletal Muscle Function but Not with Muscle Mass in Patients with Heart Failure
}

\author{
Ippei Nakano, ${ }^{1}$ MD, Shintaro Kinugawa, ${ }^{1}$ MD, Hiroaki Hori, ${ }^{2}$ MSc, Arata Fukushima, ${ }^{1}$ MD, \\ Takashi Yokota, ${ }^{1}$ MD, Shingo Takada, ${ }^{1} \mathrm{PhD}$, Naoya Kakutani, ${ }^{1,3} \mathrm{MSc}$, Yoshikuni Obata, ${ }^{1} \mathrm{MD}$, \\ Katsuma Yamanashi, ${ }^{1}$ MD and Toshihisa Anzai, ${ }^{1}$ MD
}

\begin{abstract}
Summary
Heart failure (HF) is associated with aberrant skeletal muscle impairments, which are closely linked to the severity of HF. A low level of brain-derived neurotrophic factor (BDNF), a myokine produced in the skeletal muscle, is known to be involved in reduced exercise capacity and poor prognosis in HF. However, little is known about the factors or conditions of skeletal muscle associated with BDNF levels. We investigated the association between serum BDNF levels and the skeletal muscle mass and function in HF patients $(n=60,63 \pm$ 13 years) and age-matched controls $(n=29,61 \pm 16$ years). The serum BDNF level was significantly lower in the HF patients compared to the controls $(24.9 \pm 0.9$ versus $28.6 \pm 1.3, P=0.021)$. In a univariate analysis, BDNF was significantly correlated with the peak oxygen uptake, estimated glomerular filtration rate, 10-m gait speed, and muscle strength, but not with the body mass index or lean mass in the HF group. A multiple linear regression analysis revealed that BDNF was independently associated with muscle strength ( $\beta$-coefficient $=$ 2.80, 95\% CI: $1.89-11.8, P=0.008)$. Serum BDNF levels were associated with exercise capacity and skeletal muscle function, but not with muscle mass. These novel findings may suggest that BDNF production is controlled by muscle function and activity and consequently regulates exercise capacity, highlighting the importance of adequate training regarding skeletal muscle in HF patients.
\end{abstract}

(Int Heart J 2020; 61: 96-102)

Key words: Muscle strength, Exercise capacity

$\mathrm{H}$ eart failure (HF) has become highly prevalent, characterized by reduced activities of daily living and repeated hospitalizations in addition to high mortality rates, comprising a major public health problem. ${ }^{1)}$ Aberrant skeletal muscle alterations are involved in $\mathrm{HF}^{2,3)}$ these alterations include muscle atrophy, a reduction in muscular strength, a switch in myofibers from slow type I to fast type II, and impaired energy metabolism due to a reduction in mitochondrial density and oxidative function..$^{4-6)}$ These changes have been considered important factors that lead to skeletal muscle impairments, related not only to worse symptoms but also to the severity of $\mathrm{HF}$ and the prognosis of patients with HF. ${ }^{3,7,8)}$

Brain-derived neurotropic factor (BDNF), a member of the neurotrophin family, is abundant in the hippocampus. BDNF has been reported to play a key role in various neurotrophic functions including neuroregeneration, neuroprotection, and synaptic plasticity. ${ }^{910)}$ BDNF is also present in the skeletal muscle and has been shown to be produced in response to muscle contraction. ${ }^{11)}$ Exercise training can increase the serum level of $\mathrm{BDNF}^{12)}$ and BDNF is involved in mitochondrial biogenesis and fatty acid metabolism in skeletal muscle. ${ }^{13)}$ The skeletal muscles were recently reported to secrete various cytokines and growth factors (collectively called myokines) to regulate their mass and/or function, ${ }^{14)}$ and BDNF is thus considered one of the myokines.

We reported that the serum BDNF level was decreased in HF patients compared to healthy subjects, and that the serum BDNF level was positively correlated with their peak oxygen uptake $\left(\mathrm{VO}_{2}\right){ }^{15}$ ) We and other groups also observed that decreased serum BDNF was related to all-cause cardiac death and readmission in HF patients, suggesting that the serum BDNF level could be both a less invasive biomarker that reflects the severity of HF and a predictor of prognosis in HF patients. ${ }^{16-18)}$ Using a

From the 'Department of Cardiovascular Medicine, Faculty of Medicine and Graduate School of Medicine, Hokkaido University, Sapporo, Japan, ${ }^{2}$ Department of Rehabilitation, Hokkaido University Hospital, Sapporo, Japan and ${ }^{3}$ Research Fellow of the Japan Society for the Promotion of Science.

This study was supported by grants from the Clinical Research (Medical Profession) of the Japanese Circulation Society (2017), the Japanese Association of Cardiac Rehabilitation, the Takeda Science Foundation, the Japan Ministry of Education, Science, and Culture (nos. 26350879, 15K09115, 17K10137, 19J 11266), and the Center of Innovation Program from the Japan Science and Technology Agency (JPMJCE1301).

Address for correspondence: Shintaro Kinugawa, MD, Department of Cardiovascular Medicine, Faculty of Medicine and Graduate School of Medicine, Hokkaido University, Kita-15, Nishi-7 Kita-Ku, Sapporo 060-8638, Japan. E-mail: tuckahoe@med.hokudai.ac.jp

Received for publication August 2, 2019. Revised and accepted October 25, 2019.

Released in advance online on J-STAGE January 17, 2020.

doi: 10.1536/ihj.19-400

All rights reserved by the International Heart Journal Association. 
murine model of $\mathrm{HF}$, we recently demonstrated that the administration of human recombinant BDNF improved the exercise capacity of the mice via an improvement in the mitochondrial function of their skeletal muscle. ${ }^{19)}$ The results of these clinical and basic studies indicate that BDNF is a myokine produced by the contraction of skeletal muscle, and its decrement is associated with a reduction in exercise capacity and skeletal muscle abnormalities in HF patients.

Given that skeletal muscle, i.e., muscle mass, strength and mitochondrial function is an important factor that determines exercise capacity in HF patients, ${ }^{2,20)}$ we hypothesized that the measurement of serum BDNF may show a close relationship between muscle mass and/or strength. The association between BDNF and skeletal muscle function in patients with $\mathrm{HF}$ has not been established; we therefore aimed to examine the relationship between serum BDNF and skeletal muscle mass as well as muscle strength in HF patients.

\section{Methods}

Patients and controls: Sixty Japanese patients with chronic HF (35 men and 25 women, $63 \pm 12$ years, New York Heart Association [NYHA] functional class I-III) and 29 age-matched healthy Japanese individuals as controls (19 men and 10 women, $61 \pm 14$ years) were studied. HF was diagnosed on the basis of the Framingham criteria provided in the American College of Cardiology Foundation/American Heart Association Task Force on Practice guidelines. ${ }^{21)}$ Eligible subjects were: ambulatory patients at Hokkaido University Hospital; and patients with chronic HF with NYHA class I-III with a history of one or more hospitalizations due to worsening $\mathrm{HF}$ and $\geq 1$ month post-discharge without worsening events. All patients were on standard medical therapies for $\mathrm{HF}$, and on an optimal diuretic dose.

The exclusion criteria were: patients with NYHA class IV; patients with lower limb dysfunction such as intermittent claudication and orthopedic disease; and those with significant pulmonary disease, neuromuscular disease, or disorders other than cardiac disease that limit exercise performance; patients with severe renal failure (on dialysis), severe liver failure, or infectious disease. The control group was subjects who had no history of HF and had a left ventricular ejection fraction $(\mathrm{LVEF})>50 \%$. The exclusion criteria were the same as those for the HF patients. The protocol was approved by the Medical Ethics Committee of Hokkaido University Hospital in accordance with the ethical principles described in the Declaration of Helsinki (2013 revised version), and written informed consent was obtained from all participating subjects.

Serum BDNF levels: Peripheral venous blood samples were collected in serum tubes from all subjects before cardiopulmonary exercise testing or muscle strength testing. All samples were allowed to clot and centrifuged at $1,000 \mathrm{~g}$ for 15 minutes, and then stored at $-80^{\circ} \mathrm{C}$ until analysis. The serum BDNF levels were measured by an enzyme immunoassay kit (R\&D Systems, Minneapolis, $\mathrm{MN}$ ) according to the manufacturer's protocol as described. $^{22)}$ To ensure accurate measurements, all of the samples were analyzed in duplicate by investigators blinded to the clinical information.

Assessment of muscle mass and strength: We measured the subjects' body composition by using a Discovery DXA system (Discovery-A, Hologic, Marlborough, MA). The values of total and segmental mass, lean mass, fat mass, and bone masses were recorded. The lean mass was used to estimate the muscle mass. ${ }^{23)}$ The muscle strength of the quadriceps femoris muscle was evaluated by an isokinetic dynamometer (Biodex System 4, Biodex Medical Systems, Shirley, NY) as described. ${ }^{24}$ To obtain the isokinetic peak torque, the subject performed 10 dynamic repetitions of concentric knee extensions/flexions at $180 \%$ second each with the right and left legs separately at maximal effort. The highest value of the torque $(\mathrm{Nm})$ recorded during the unilateral knee extension was defined as the muscle strength.

Cardiopulmonary exercise testing: Cardiopulmonary exercise testing was performed using an upright electromechanical bicycle ergometer (Aerobike 75XLII, Combi Wellness, Tokyo) with a ramp protocol as described. ${ }^{25}$ \{Yokota, 2011 \#1368; Yokota, 2011 \#112\} Briefly, after 3 minutes of unloaded cycling, the exercise load was increased continually in $10-15 \mathrm{~W} /$ minute increments in the $\mathrm{HF}$ patients and 20-25 W/minute increments in the control subjects. The subjects stopped the exercise when they had dyspnea and/or severe leg fatigue. The $\mathrm{VO}_{2}$ was obtained by a breath-by-breath method throughout the examination by an expired gas analyzer (Aeromonitor AE-300S, Minato Medical Science, Osaka, Japan). Peak $\mathrm{VO}_{2}$ was defined as the $\mathrm{VO}_{2}$ attained at maximal exercise, and the anaerobic threshold (AT) was determined by the V-slope method.

Other clinical variables: Body weight and height were measured, and the body mass index (BMI) (body weight/ [height $]^{2}, \mathrm{~kg} / \mathrm{m}^{2}$ ) was calculated. The etiology of $\mathrm{HF}$ and the medication(s) used by each patient were determined based on the medical records. The 10-m gait speed was measured based on the duration of the time required to walk the middle $10 \mathrm{~m}$ during a maximum $14 \mathrm{~m}$-walk.

Left ventricular (LV) end-diastolic dimension (EDD) and end-systolic dimension (ESD) were measured in the parasternal long-axis view by transthoracic echocardiography. The LV ejection fraction (LVEF) was calculated by the modified Simpson's method from the apical 4- and 2chamber views. All subjects underwent measurements of hemoglobin, platelets, estimate glomerular filtration rate (eGFR), hemoglobin A1c (HbA1c), and plasma brain natriuretic peptide (BNP). The eGFR was calculated from the serum creatinine values and age, using the Japanese equation.

Statistical analyses: The necessary sample sizes of patients were calculated based on the study by Fukushima, et $a l .{ }^{15)}$ To detect the effect compared with the threshold change of 0 under the conditions of $\alpha=0.05, \beta=0.2$ and the allocation ratio $=2(\mathrm{HF} /$ controls $)$, the necessary sample sizes calculated were $n=51$ for the HF group and $n=$ 25 for the controls. The results are expressed as the mean $\pm \mathrm{SD}$ for continuous variables and as numbers and percentages for categorical variables. We used the unpaired Student $t$-test or the Mann-Whitney $U$-test to compare 
Table I. Characteristics of the Control Subjects and Patients with HF

\begin{tabular}{|c|c|c|c|}
\hline & Control $(n=29)$ & $\mathrm{HF}(n=60)$ & $P$-value \\
\hline \multicolumn{4}{|l|}{ Demographic and clinical factors } \\
\hline Age, years & $61 \pm 16$ & $63 \pm 13$ & 0.632 \\
\hline Male, $n(\%)$ & $19(66)$ & $35(58)$ & 0.514 \\
\hline BMI, $\mathrm{kg} / \mathrm{m}^{2}$ & $24.9 \pm 3.2$ & $23.3 \pm 3.7$ & 0.049 \\
\hline Hypertension, $n(\%)$ & $13(45)$ & $18(30)$ & 0.169 \\
\hline Diabetes mellitus, $n(\%)$ & $7(24)$ & $15(25)$ & 0.930 \\
\hline Dyslipidemia, $n(\%)$ & $16(55)$ & $38(63)$ & 0.460 \\
\hline NYHA: I / II / III & & $8 / 44 / 8$ & \\
\hline $\mathrm{HFpEF}, n(\%)$ & & $13(22)$ & \\
\hline \multicolumn{4}{|l|}{ Medication, $n(\%)$} \\
\hline ACE-Is or ARBs & $8(28)$ & $58(97)$ & $<0.001$ \\
\hline Aldosterone antagonists & $3(10)$ & $37(62)$ & $<0.001$ \\
\hline$\beta$-blockers & $4(14)$ & $55(92)$ & $<0.001$ \\
\hline Diuretics & $1(3)$ & $39(65)$ & $<0.001$ \\
\hline Statins & $14(48)$ & $38(63)$ & 0.177 \\
\hline \multicolumn{4}{|l|}{ Etiology of HF, $n(\%)$} \\
\hline Ischemic heart disease & - & $18(30)$ & \\
\hline Non-ischemic heart disease & - & $42(70)$ & \\
\hline \multicolumn{4}{|l|}{ Laboratory test results } \\
\hline Hemoglobin, g/dL & $14.3 \pm 1.4$ & $13.3 \pm 1.4$ & 0.001 \\
\hline Platelets, $\times 10^{4} / \mu \mathrm{L}$ & $215 \pm 51$ & $196 \pm 51$ & 0.097 \\
\hline eGFR, mL/minute $/ 1.73 \mathrm{~m}^{2}$ & $76.2 \pm 18.0$ & $52.3 \pm 18.9$ & $<0.001$ \\
\hline $\mathrm{HbAlc}, \%$ & $6.0 \pm 0.7$ & $6.0 \pm 0.7$ & 0.936 \\
\hline Log BNP, pg/mL & $1.1 \pm 0.1$ & $2.0 \pm 0.1$ & $<0.001$ \\
\hline Serum BDNF, ng/mL & $28.6 \pm 7.0$ & $24.9 \pm 6.8$ & 0.021 \\
\hline \multicolumn{4}{|l|}{ Echocardiographic parameters } \\
\hline LVEDD, $\mathrm{mm}$ & $47.5 \pm 3.2$ & $60.5 \pm 11.2$ & $<0.001$ \\
\hline LVESD, mm & $30.1 \pm 2.2$ & $49.9 \pm 14.6$ & $<0.001$ \\
\hline LVEF, \% & $65.3 \pm 4.7$ & $38.1 \pm 14.2$ & $<0.001$ \\
\hline \multicolumn{4}{|l|}{ Cardiopulmonary exercise test } \\
\hline Peak $\mathrm{VO}_{2}, \mathrm{~mL} / \mathrm{kg} /$ minute & $24.8 \pm 6.6$ & $16.8 \pm 4.3$ & $<0.001$ \\
\hline $\mathrm{AT}, \mathrm{mL} / \mathrm{kg} / \mathrm{minute}$ & $13.9 \pm 2.8$ & $10.2 \pm 2.2$ & $<0.001$ \\
\hline Peak respiratory exchange ratio & $1.23 \pm 0.08$ & $1.20 \pm 0.09$ & 0.267 \\
\hline \multicolumn{4}{|l|}{ Skeletal muscle findings } \\
\hline Lean mass, $\mathrm{kg}$ & $49.3 \pm 9.8$ & $41.7 \pm 7.5$ & 0.004 \\
\hline 10-m gait speed, seconds & $5.4 \pm 1.1$ & $6.1 \pm 1.4$ & 0.023 \\
\hline Muscle strength, $\mathrm{Nm}$ & $101.8 \pm 29.9$ & $84.5 \pm 22.9$ & 0.004 \\
\hline
\end{tabular}

Data are mean \pm SD. ACE-I indicates angiotensin-converting enzyme inhibitor; ARB, angiotensin receptor blocker; AT, anaerobic threshold; BDNF, brain-derived neurotrophic factor; BMI, body mass index; BNP, brain natriuretic peptide; EDD, end-diastolic diameter; EF, ejection fraction; ESD, end-systolic diameter; eGFR, estimated glomerular filtration rate; $\mathrm{Hb}$, hemoglobin; $\mathrm{HF}$, heart failure; $\mathrm{HFpEF}$, HF with preserved EF; LV, left ventricular; MVC, maximum voluntary contraction; NYHA, New York Heart Association function class; and $\mathrm{VO}_{2}$, oxygen uptake.

continuous variables, and the chi-square test for categorical variables. A univariate linear regression model was used to determine the correlations between the serum BDNF levels and other clinical variables.

Multiple linear regression analysis was conducted to identify the independent variables associated with serum BDNF levels. Clinical parameters or variables with a $P$ value $<0.2$ in the univariate model were considered for inclusion. All analyses were performed using JMP 14.0.0 software (SAS, Cary, NC). The differences were considered significant when the $P$-values were $<0.05$.

\section{Results}

Patient characteristics: The age, gender, and past history of the HF and control groups were comparable, whereas the BMI was significantly lower in the HF group ( $P=$ 0.049) (Table I). Regarding the NYHA functional class, 8 patients were in class I, 44 class II, and 8 class III. The etiologies of HF were ischemic heart disease in 18 patients and non-ischemic heart disease in the other 42 patients. Thirteen patients $(22 \%)$ had HF with preserved EF (HFpEF), and the other 48 patients had HF with reduced $\mathrm{EF}$ (HFrEF). Angiotensin-converting enzyme inhibitors or angiotensin II type I receptor antagonists were used in $97 \%$ of the HF patients; $\beta$-blockers in $92 \%$, aldosterone antagonists in $62 \%$, and diuretics in $63 \%$.

In the echocardiography, the HF patients had significantly greater LVEDD and significantly lower LVEF values (both $P<0.001$ ) compared to the control group. The peak $\mathrm{VO}_{2}$ and $\mathrm{AT}$ values were significantly lower in the HF patients (both $P<0.001$ ). The plasma BNP values 


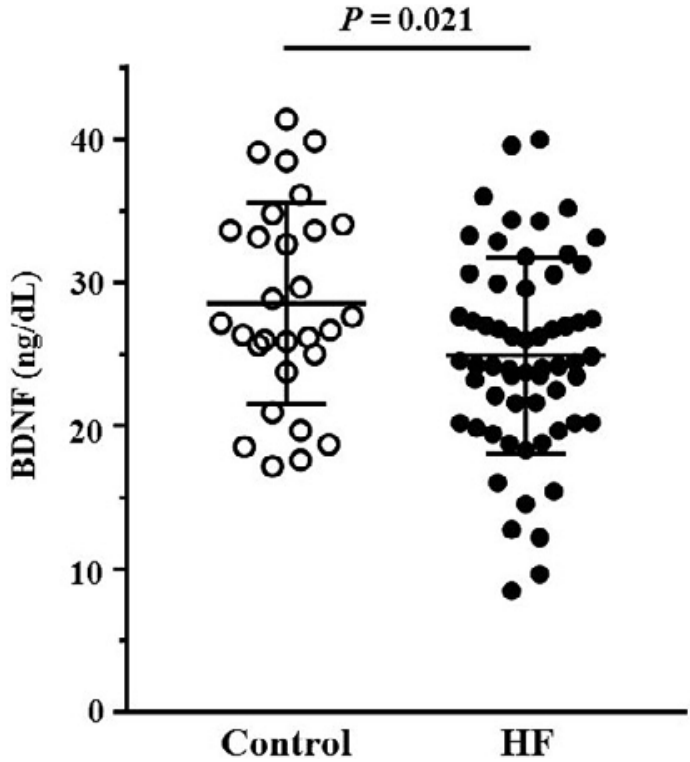

Figure 1. Serum BDNF levels in the control subjects (open circles, $n=29$ ) and patients with HF (closed circles, $n=60$ ).

were significantly higher and the hemoglobin and eGFR values were significantly lower in the HF patients compared to the control subjects (both $P<0.001$ ). The values of lean mass, $10-\mathrm{m}$ gait speed, and muscle strength were significantly lower in the HF group.

Serum BDNF levels: The serum BDNF levels were significantly lower in the HF patients compared to the control subjects $(24.9 \pm 0.9$ versus $28.6 \pm 1.3 \mathrm{ng} / \mathrm{mL}, P=$ 0.021) (Figure 1). Among $60 \mathrm{HF}$ patients, the BDNF levels did not differ between the males and females $(P=$ 0.939 ) or between other clinical variables, including diabetic and non-diabetic patients $(P=0.882)$. In addition, the BDNF levels did not differ between the HFpEF and HFrEF patients $(P=0.152)$ (data not shown).

Correlation between serum BDNF and clinical variables in the HF patients: In the univariate analysis, the serum BDNF levels of the HF patients were significantly correlated with their values of peak $\mathrm{VO}_{2}(r=0.372, P=$ $0.007)$, AT $(r=0.372, P=0.006)$, hemoglobin $(r=0.412$, $P=0.001)$, eGFR $(r=0.305, P=0.018), 10-\mathrm{m}$ gait speed $(r=-0.368, P=0.005)$, and muscle strength $(r=$ $0.342, P=0.009)$, but not with their age, BMI, lean mass, LVEDD, LVEF, HbA1c, or the plasma log BNP level (Table II, Figures 2, 3).

In the multiple analysis, muscle strength $(\beta$ coefficient $=2.80,95 \% \mathrm{CI}: 1.89-11.8, P=0.008)$ was identified as an independent determinant of the serum BDNF level, conditional upon the other predictors (Table III).

\section{Discussion}

The main findings of the present study are that (1) the serum BDNF level was lower in HF patients compared to the control subjects, and it was correlated with exercise capacity, and (2) the serum BDNF level in the
Table II. Univariate Linear Model of BDNF in the HF Group

\begin{tabular}{lcc}
\hline \multicolumn{1}{c}{ Variable } & $\begin{array}{c}\text { Univariate correlation } \\
\text { coefficient }\end{array}$ & $P$-value \\
\hline Age, years & 0.018 & 0.891 \\
BMI, kg/m² & -0.001 & 0.994 \\
$\mathrm{LVEDD}, \mathrm{mm}$ & -0.103 & 0.434 \\
$\mathrm{LVEF}, \%$ & 0.174 & 0.185 \\
Peak VO, $\mathrm{mL} / \mathrm{kg} / \mathrm{minute}$ & 0.321 & 0.019 \\
$\mathrm{AT}, \mathrm{mL} / \mathrm{kg} / \mathrm{minute}$ & 0.372 & 0.006 \\
Hemoglobin, g/dL & 0.412 & 0.001 \\
eGFR, mL/minute/1.73 m ${ }^{2}$ & 0.305 & 0.018 \\
Log BNP, pg/mL & -0.176 & 0.186 \\
HbA1c, \% & -0.143 & 0.278 \\
Lean mass, kg & 0.018 & 0.907 \\
10-m gait speed, sec & -0.368 & 0.005 \\
Muscle strength, Nm & 0.342 & 0.009 \\
\hline Abbreviations are explained in the Table I footnote and the text.
\end{tabular}

HF patients was significantly correlated with the muscle function measurements, but not with the BMI or lean mass.

We also observed that the serum BDNF level was correlated with aspects of exercise capacity, i.e., the peak $\mathrm{VO}_{2}$ and AT. This result is concordant with our previous findings ${ }^{15,16)}$ as well as reports from other groups. ${ }^{17,26)}$ Interestingly, the serum BDNF levels of the HF patients in the present study were higher compared to those in previous reports. This difference may be due to the severity of HF of the enrolled subjects: $87 \%$ of the HF patients in this study were NYHA class I/II and $13 \%$ were NYHA class III, with a mean peak $\mathrm{VO}_{2}$ of $16.8 \mathrm{~mL} / \mathrm{kg} /$ minute, and our HF group showed a mean BDNF level of $24.9 \mathrm{ng} / \mathrm{mL}$. Our previous study ${ }^{15)}$ with a mean BDNF level at $16.6 \mathrm{ng}$ / $\mathrm{mL}$ examined HF patients with more severe $\mathrm{HF}$ and lower exercise intolerance: $76 \%$ of that patient series was NYHA class I/II and $24 \%$ was NYHA class III, and the mean peak $\mathrm{VO}_{2}$ was $14.0 \mathrm{~mL} / \mathrm{kg} /$ minute. Kadowaki, et al reported the mean BDNF level of $14.7 \mathrm{ng} / \mathrm{mL}$ in $\mathrm{HF}$ patients, $53 \%$ of whom were NYHA class II and $47 \%$ of whom were NYHA III/IV, including patients with decompensated $\mathrm{HF}^{26)}$ Therefore, serum BDNF is present at lower values in patient groups with a higher severity of $\mathrm{HF}$, and together with the finding that lower serum BDNF is associated with exercise intolerance and worse prognosis in HF patients, ${ }^{15,16}$ these results indicate that the serum BDNF level well reflects the severity of HF.

In HF, aberrant skeletal muscle impairments are known to occur both in quantity and quality, ${ }^{4,6,27)}$ and these changes have been considered important factors of exercise intolerance in HF patients. We have reported the association between serum BDNF and exercise intolerance; however, the relationship between BDNF and skeletal muscle mass and/or function remained unclear. The present study revealed for the first time that serum BDNF shows correlations with skeletal muscle function such as the 10-m gait speed and muscle strength, but not with the BMI or lean mass. Tsai, et al reported similarly that exercise training increased the serum BDNF levels in type 2 diabetic patients, and they noted that the serum BDNF levels were correlated with the changes of muscle 
$\mathbf{A}$

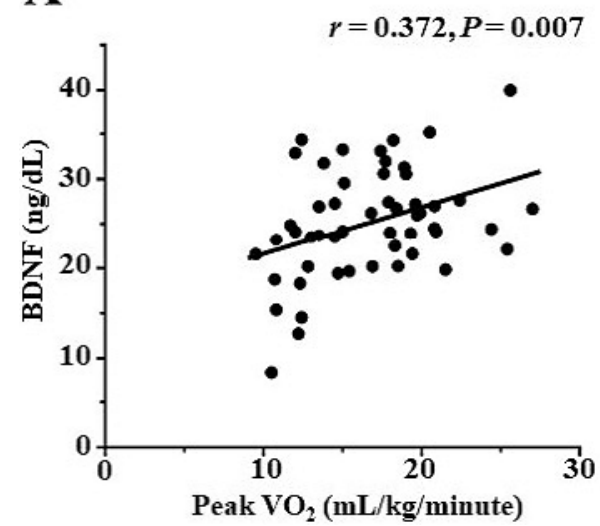

B

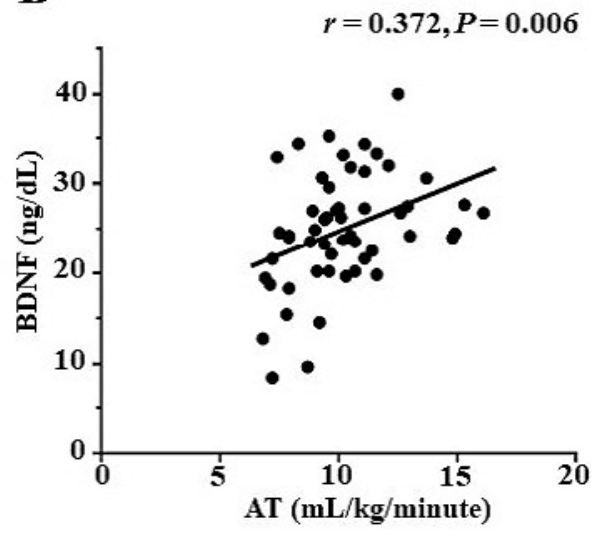

Figure 2. Correlation between the serum BDNF level and peak $\mathrm{VO}_{2}(\mathbf{A})$, and $\mathrm{AT}(\mathbf{B})$ in the HF patients.

A

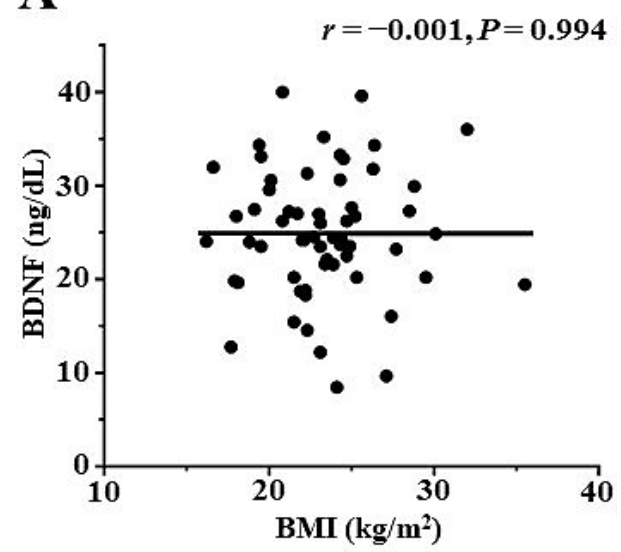

C

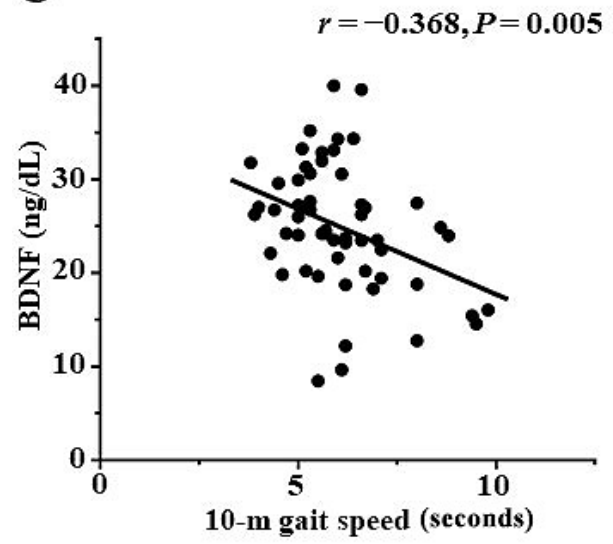

B

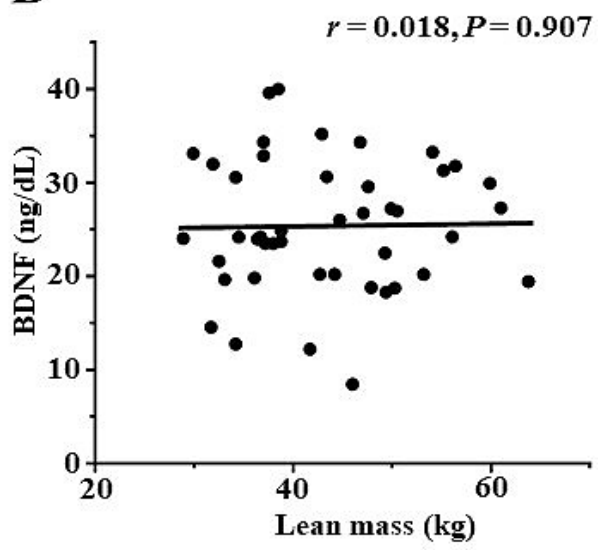

D

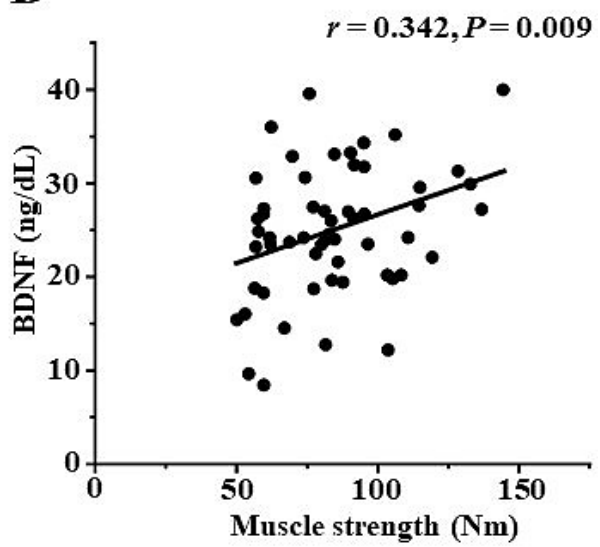

Figure 3. Correlation between the serum BDNF level and the BMI (A), lean mass $(\mathbf{B}), 10-\mathrm{m}$ gait speed $(\mathbf{C})$, and muscle strength (D) in the HF patients.

strength, but not with the BMI. ${ }^{28)}$ Accordingly, these results suggest that muscle function or activity, rather than muscle mass, is important for the increase in BDNF levels.

BDNF seems to play a key role in mediating the benefits of exercise. It has been well documented that ex- ercise increases the production of BDNF. For example, exercise increases BDNF levels in the hippocampus, contributing to improvements in cognitive function. ${ }^{29-31)} \mathrm{Sev}-$ eral other studies revealed that acute aerobic exercise and endurance training also increase serum BDNF. ${ }^{32)}$ However, the source of the increased circulating BDNF remains to 
Table III. Multiple Regression Analysis of BDNF and Other Clinical Variables in the HF Group

\begin{tabular}{lccc}
\hline \multicolumn{1}{c}{ Variable } & $\begin{array}{c}\text { Correlation } \\
\text { coefficient }\end{array}$ & $95 \% \mathrm{CI}$ & $P$-value \\
\hline Age, years & 3.67 & $-1.72-9.07$ & 0.175 \\
Male (0) & -1.16 & $-4.97-2.65$ & 0.541 \\
LVEF, \% & -1.89 & $-2.66-6.43$ & 0.406 \\
Log BNP, pg/mL & -0.31 & $-4.89-4.27$ & 0.892 \\
Lean mass, kg & 1.44 & $-5.68-8.56$ & 0.684 \\
Muscle strength, Nm & 6.85 & $1.89-11.8$ & 0.008 \\
\hline Male and female were assigned values of 0 and 1, respectively.
\end{tabular}

be elucidated. Several possibilities are proposed; first, most of the circulating BDNF is stored in platelets, and its activated release from platelets in response to exercise might contribute to the increased level of BDNF. ${ }^{33)}$ Second, the brain might be another source, since cerebral BDNF is known to pass through the blood-brain barrier, ${ }^{34)}$ and preliminary evidence indicated that $70 \%-80 \%$ of the increase in BDNF due to exercise is derived from the brain. ${ }^{35)}$

BDNF was also shown to be synthesized by skeletal muscle in response to contraction and exercise. ${ }^{11,36,37)}$ Indeed, Matthews, et al demonstrated that skeletal muscle cells themselves produce BDNF in response to contraction. ${ }^{11)}$ However, they also observed that the elevation of skeletal-muscle BDNF did not correlate with serum BDNF, suggesting that skeletal muscle BDNF does not transfer into circulation and acts locally, enhancing fatty acid oxidation in the muscle, at least in the acute phase after exercise. In the chronic phase, the role of BDNF in skeletal muscle post-exercise was reported to be more than the regulation of energy metabolism. ${ }^{38)}$

Our present findings indicate that in conditions of chronic skeletal muscle impairments (as in the setting of $\mathrm{HF}$ ), it is possible that BDNF production from skeletal muscle is involved in the serum BDNF level, and that muscle activity is important for the increase in serum BDNF, since the muscle function was more closely associated with BDNF compared to the muscle mass. However, more evidence is needed to evaluate the source of BDNF in HF patients.

BDNF is known to affect exercise capacity by regulating skeletal muscle mitochondrial function and content, but the cause-and-result relationship between BDNF and muscle function is not clear. BDNF was shown to activate the AMP-activated protein kinase (AMPK) and enhance fatty acid oxidation, ${ }^{11)}$ and it triggers the AMPK/CREB/ PGC- $1 \alpha$ pathways to increase cellular respiration by promoting mitochondrial biogenesis in skeletal muscle. ${ }^{13)}$ The depletion of BDNF in $\mathrm{C} 2 \mathrm{C} 12$ myoblasts decreased the level of phosphorylation of AMPK and PGC- $1 \alpha$ proteins. ${ }^{39)}$ We recently reported that BDNF was reduced in the skeletal muscle of a murine model of HF after myocardial infarction, and that the administration of human recombinant BDNF ameliorated the reduction of the endurance exercise capacity and improved the mitochondrial respiration of the skeletal muscle. ${ }^{19)}$

Taken together, all of these findings indicate that BDNF regulates mitochondrial function through AMPK/
PGC- $1 \alpha$ and affects the endurance exercise capacity. To date, there is no evidence showing that BDNF directly regulates muscle mass or function. Based on the present results, it is plausible that the intensity of muscle function regulates the production of $\mathrm{BDNF}$, rather than that BDNF controls muscle function. Further elucidation of the underlying mechanisms is needed.

Elderly patients with HF have a high prevalence of sarcopenia, which is a state of muscle impairment in both mass and function. ${ }^{5)}$ Sarcopenia is an independent prognostic factor in $\mathrm{HF}^{40)}$ but it has been a great concern whether the exercise intolerance is mainly due to muscle dysfunction or the decrease in muscle mass considering the pathophysiology of HF patients. Exercise training is beneficial for $\mathrm{HF}$ patients with sarcopenia, but muscle training generally improves muscle strength in advance of an improvement in muscle mass, ${ }^{41)}$ making it difficult for such patients to gain muscle mass. In light of our present findings, it appears that the improvement in muscle function may increase myokines (including BDNF) and may contribute to the multiple health benefits associated with exercise, providing a significant impact in clinical practice.

The present study revealed for the first time that serum BDNF was associated with skeletal muscle function, but not with muscle mass. We speculate that BDNF is secreted by muscle activity, regulates mitochondrial function in the skeletal muscle, and defines exercise endurance. This novel finding may provide a crucial clue for future therapies, such as medications for HF-related muscle impairments.

There are several limitations of this study that should be acknowledged. First, we cannot make a conclusion regarding the causal relationship between BDNF and muscle function in this cross-sectional observation with a relatively small number of patients, and thus a large portion of the mechanism remains speculative. Further investigations and large-population studies are warranted. Second, our HF group was comprised of both HFpEF and HFrEF patients. Although the BDNF levels did not differ between the groups, we did not compare further group values due to the limited sample size. Finally, the source of the circulating BDNF is not clear at present. Although evidence shows BDNF production in skeletal muscle by contraction, the precise roles of muscle and serum BDNF remain to be elucidated.

\section{Disclosure}

Conflicts of interest: The authors state that there are no conflicts of interest to declare.

\section{References}

1. Roger VL. Epidemiology of heart failure. Circ Res 2013; 113: 646-59.

2. Tucker WJ, Haykowsky MJ, Seo Y, Stehling E, Forman DE. Impaired exercise tolerance in heart failure: Role of skeletal muscle morphology and function. Curr Heart Fail Rep 2018; 15: 323-31. 
3. Coats AJ, Clark AL, Piepoli M, Volterrani M, Poole-Wilson PA Symptoms and quality of life in heart failure: The muscle hypothesis. Br Heart J 1994; 72: S36-9.

4. Mancini DM, Coyle E, Coggan A, et al. Contribution of intrinsic skeletal muscle changes to 31P NMR skeletal muscle metabolic abnormalities in patients with chronic heart failure. Circulation 1989; 80: 1338-46.

5. Fulster S, Tacke M, Sandek A, et al. Muscle wasting in patients with chronic heart failure: results from the studies investigating co-morbidities aggravating heart failure (SICA-HF). Eur Heart J 2013; 34: 512-9.

6. Kinugawa S, Takada S, Matsushima S, Okita K, Tsutsui H. Skeletal muscle abnormalities in heart failure. Int Heart J 2015; 56: 475-84.

7. Mancini DM, Eisen H, Kussmaul W, Mull R, Edmunds LH Jr., Wilson JR. Value of peak exercise oxygen consumption for optimal timing of cardiac transplantation in ambulatory patients with heart failure. Circulation 1991; 83: 778-86.

8. Clark AL, Poole-Wilson PA, Coats AJ. Exercise limitation in chronic heart failure: Central role of the periphery. J Am Coll Cardiol 1996; 28: 1092-102.

9. Lewin GR, Barde YA. Physiology of the neurotrophins. Annu Rev Neurosci 1996; 19: 289-317.

10. Mattson MP, Maudsley S, Martin B. BDNF and 5-HT: A dynamic duo in age-related neuronal plasticity and neurodegenerative disorders. Trends Neurosci 2004; 27: 589-94.

11. Matthews VB, Astrom MB, Chan MH, et al. Brain-derived neurotrophic factor is produced by skeletal muscle cells in response to contraction and enhances fat oxidation via activation of AMP-activated protein kinase. Diabetologia 2009; 52: 1409-18.

12. Ferris LT, Williams JS, Shen CL. The effect of acute exercise on serum brain-derived neurotrophic factor levels and cognitive function. Med Sci Sports Exerc 2007; 39: 728-34.

13. Wood J, Tse MCL, Yang X, et al. BDNF mimetic alleviates body weight gain in obese mice by enhancing mitochondrial biogenesis in skeletal muscle. Metabolism 2018; 87: 113-22.

14. Pedersen BK, Febbraio MA. Muscles, exercise and obesity: Skeletal muscle as a secretory organ. Nat Rev Endocrinol 2012; 8: 457-65.

15. Fukushima A, Kinugawa S, Homma T, et al. Decreased serum brain-derived neurotrophic factor levels are correlated with exercise intolerance in patients with heart failure. Int $\mathrm{J}$ Cardiol 2013; 168: e142-4.

16. Fukushima A, Kinugawa S, Homma $\mathrm{T}$, et al. Serum brainderived neurotropic factor level predicts adverse clinical outcomes in patients with heart failure. J Card Fail 2015; 21: 3006.

17. Shibata A, Hanatani A, Izumi Y, Kitada R, Iwata S, Yoshiyama M. Serum brain-derived neurotrophic factor level and exercise tolerance complement each other in predicting the prognosis of patients with heart failure. Heart Vessels 2018; 33: 1325-33.

18. Takashio S, Sugiyama S, Yamamuro M, et al. Significance of low plasma levels of brain-derived neurotrophic factor in patients with heart failure. Am J Cardiol 2015; 116: 243-9.

19. Matsumoto J, Takada S, Kinugawa S, et al. Brain-derived neurotrophic factor improves limited exercise capacity in mice with heart failure. Circulation 2018; 138: 2064-6.

20. van der Zwaard S, de Ruiter CJ, Noordhof DA, et al. Maximal oxygen uptake is proportional to muscle fiber oxidative capacity, from chronic heart failure patients to professional cyclists. J Appl Physiol (1985) 2016; 121: 636-45.

21. Yancy CW, Jessup M, Bozkurt B, et al. 2013 ACCF/AHA guideline for the management of heart failure: A report of the American College of Cardiology Foundation/American Heart Association Task Force on Practice Guidelines. J Am Coll Cardiol 2013; 62: e147-239.

22. Laske C, Stransky E, Leyhe T, et al. Stage-dependent BDNF se- rum concentrations in Alzheimer's disease. J Neural Transm (Vienna) 2006; 113: 1217-24.

23. Chen Z, Wang Z, Lohman T, et al. Dual-energy X-ray absorptiometry is a valid tool for assessing skeletal muscle mass in older women. J Nutr 2007; 137: 2775-80.

24. Maughan RJ, Watson JS, Weir J. Strength and cross-sectional area of human skeletal muscle. J Physiol 1983; 338: 37-49.

25. Kakutani N, Fukushima A, Yokota T, et al. Impact of high respiratory exchange ratio during submaximal exercise on adverse clinical outcome in heart failure. Circ J 2018; 82: 2753-60. (doi: 10.1253/circj.CJ-18-0103).

26. Kadowaki S, Shishido T, Honda Y, et al. Additive clinical value of serum brain-derived neurotrophic factor for prediction of chronic heart failure outcome. Heart Vessels 2016; 31: 535-44.

27. Fulster S, Tacke M, Sandek A, et al. Muscle wasting in patients with chronic heart failure: Results from the studies investigating co-morbidities aggravating heart failure (SICA-HF). Eur Heart J 2013; 34: 512-9.

28. Tsai SW, Chan YC, Liang F, Hsu CY, Lee IT. Brain-derived neurotrophic factor correlated with muscle strength in subjects undergoing stationary bicycle exercise training. J Diabetes Complications 2015 ; 29: 367-71.

29. Abeer ED, Doaa MS, Fatma ID. Impact of exercise and vitamin B1 intake on hippocampal brain-derived neurotrophic factor and spatial memory performance in a rat model of stress. J Nutr Sci Vitaminol 2015; 61: 1-7.

30. Wrann CD, White JP, Salogiannnis J, et al. Exercise induces hippocampal BDNF through a PGC-1alpha/FNDC5 pathway. Cell Metab 2013; 18: 649-59.

31. Kim YM, Ji ES, Kim SH, et al. Treadmill exercise improves short-term memory by enhancing hippocampal cell proliferation in quinolinic acid-induced Huntington's disease rats. J Exerc Rehabil 2015; 11: 5-11.

32. Huang T, Larsen KT, Ried-Larsen M, Moller NC, Andersen LB. The effects of physical activity and exercise on brain-derived neurotrophic factor in healthy humans: A review. Scand J Med Sci Sports 2014; 24: 1-10.

33. Tang SW, Chu E, Hui T, Helmeste D, Law C. Influence of exercise on serum brain-derived neurotrophic factor concentrations in healthy human subjects. Neurosci Lett 2008; 431: 62-5.

34. Pan W, Banks WA, Fasold MB, Bluth J, Kastin AJ. Transport of brain-derived neurotrophic factor across the blood-brain barrier. Neuropharmacology 1998; 37: 1553-61.

35. Rasmussen P, Brassard P, Adser H, et al. Evidence for a release of brain-derived neurotrophic factor from the brain during exercise. Exp Physiol 2009; 94: 1062-9.

36. Hurtado E, Cilleros V, Nadal L, et al. Muscle contraction regulates BDNF/TrkB signaling to modulate synaptic function through presynaptic cPKCalpha and cPKCbetaI. Front Mol Neurosci 2017; 10: 147

37. Lee HW, Ahmad M, Wang HW, Leenen FH. Effects of exercise training on brain-derived neurotrophic factor in skeletal muscle and heart of rats post myocardial infarction. Exp Physiol 2017; 102: $314-28$

38. Yu T, Chang Y, Gao XL, Li H, Zhao P. Dynamic expression and the role of BDNF in exercise-induced skeletal muscle regeneration. Int J Sports Med 2017; 38: 959-66.

39. Zhang Z, Wang B, Fei A. BDNF contributes to the skeletal muscle anti-atrophic effect of exercise training through AMPK-PGC 1alpha signaling in heart failure mice. Arch Med Sci 2019; 15: 214-22.

40. Onoue Y, Izumiya Y, Hanatani S, et al. A simple sarcopenia screening test predicts future adverse events in patients with heart failure. Int J Cardiol 2016; 215: 301-6.

41. Borde R, Hortobagyi T, Granacher U. Dose-response relationships of resistance training in healthy old adults: A systematic review and meta-analysis. Sports Med 2015; 45: 1693-720. 